\title{
High-density data recording via laser thermo-lithography and ion-beam etching
}

\author{
I.V. Gorbov ${ }^{1}$, A.A. Kryuchyn ${ }^{1}$, K.P. Grytsenko², D.Yu. Manko', Yu.O. Borodin ${ }^{1}$ \\ ${ }^{I}$ Institute for Information Recording of National Academy of Science of Ukraine \\ 2, str. M. Shpaka, 03113, Kiev, Ukraine \\ ${ }^{2} V$. Lashkaryov Institute of Semiconductor Physics of NAS of Ukraine, 41, prospect Nauky, 03028 Kiev, Ukraine \\ Gorbov I.V.: tel.: (38-044) 454-22-19, fax: (38-044) 456-33-18, e-mail: ivan-gorbov@list.ru \\ Kryuchyn A.A.: tel.: (38-044) 454-21-52,fax: (38-044) 241-72-33, e-mail: ipri@ipri.kiev.ua \\ Grytsenko K.P.: tel.: (38-044) 525-55-30,e-mail: d.grytsenko@gmail.com \\ Manko D.Yu.: tel.: (38-044) 454-22-09, fax: (38-044) 456-33-18, e-mail: dmitriy.manko@gmail.com \\ Borodin Yu.O.: tel.: (38-044) 454-21-14, fax: (38-044) 456-33-18, e-mail: borodiny@yahoo.com
}

\begin{abstract}
Pits $250-300-\mathrm{nm}$ wide were obtained on the surface of thin organic nanocomposite film using master-disc laser-burning station with $405 \mathrm{~nm}$ laser beam focused by $0.85 \mathrm{NA}$ lens. The film with obtained pits was used as a mask for subsequent reactive ion-beam etching of glass substrate. Finally, $150-200-\mathrm{nm}$ pits were performed on the substrate surface. Nanocomposite films were based on organic positive photoresist with a dye inclusions. This dye is characterized by wide absorption band within the spectral region 390-410 $\mathrm{nm}$ and can be evaporated by laser irradiation with the wavelength $405 \mathrm{~nm}$.
\end{abstract}

Keywords: optical data recording, laser thermo-lithography, organic nanocomposite films, ion-beam etching.

Manuscript received 11.12.13; revised version received 23.01.14; accepted for publication 20.03.14; published online 31.03.14.

\section{Introduction}

A recognized advantage of optical storage is the low cost mass-production of read-only-memory media [1]. Generally, optical data recording is the process where one or several features of recording medium are changing under the influence of focused laser radiation. In conventional photochemical data recoding, all irradiated media are exposed and changed. In this case, the data recording density is restricted by the diffraction limit. Using the Rayleigh criterion that is determined by the relationship $\mathrm{d}_{\min }=0.61 \times \lambda / \mathrm{NA}$ (where $\lambda$ is the wavelength, NA - numerical aperture, $\mathrm{d}_{\min }-$ minimal size of a pit), we can obtain that for $\lambda=405 \mathrm{~nm}$, $\mathrm{NA}=0.85, \quad \mathrm{~d}_{\min } \sim 0.3 \mu \mathrm{m} ; \quad$ and for $\lambda=375 \mathrm{~nm}$, $\mathrm{NA}=0.95, \mathrm{~d}_{\min }=0.2 \mu \mathrm{m}$.

To reach these sizes, we usually use photochemical process that is widely used in optical disc mastering. The process is described for recording and reproducing information comprising the steps of imagewise exposing the recording layer including a polymeric composition that is photochemically hardenable under actinic light and treating the exposed layer with heat and/or a vapor containing solvent for the said polymeric composition.

The development of optical data storage has reached a stage when further progress along the classical way of reducing the diffraction limit seems questionable. In this sense, a parallel between optical data storage and high-resolution lithography is evident. However, because of the professional environment in which optical lithography is used, exotic wavelengths like $193 \mathrm{~nm}$ (KrF-laser) or even $13.5 \mathrm{~nm}$ (plasma-generated extreme UV) are possible. Liquid immersion has also been successfully applied in optical lithography to further increase the capacity of integrated circuits [2]. These 


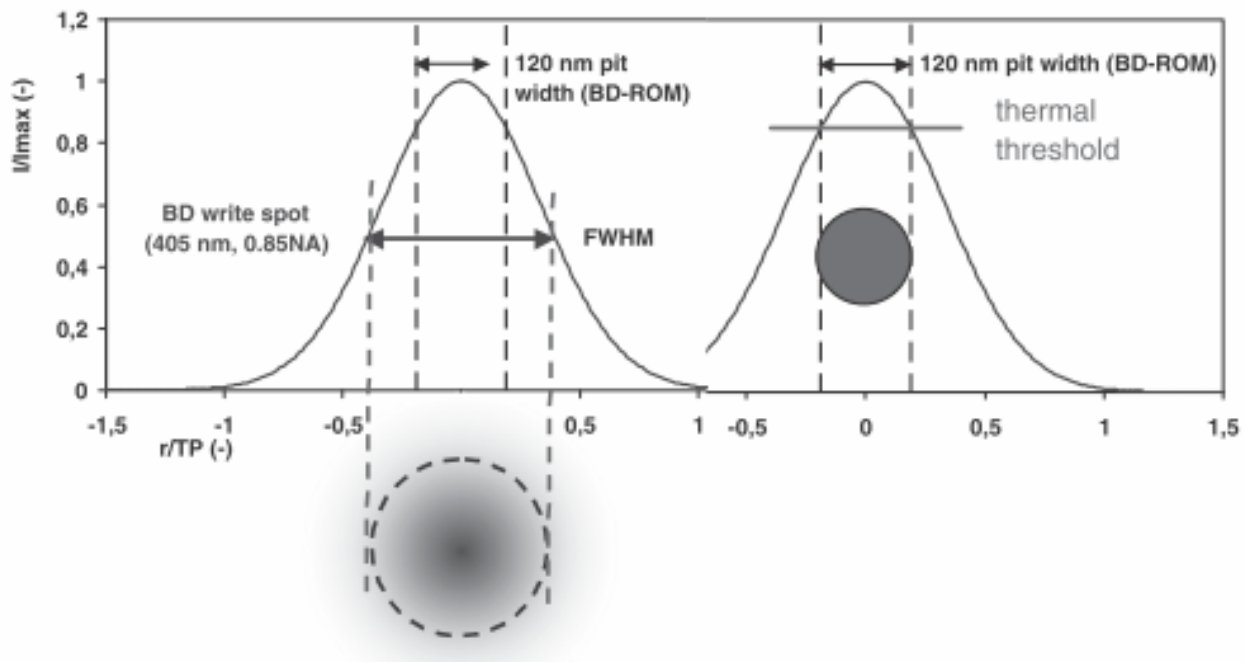

Fig. 1 Schematic view of the intensity profile for a blue mastering spot (405 nm, NA=0.9) and the target BD-ROM pit width [3].

technologies are expensive and complicate. In our opinion, if a suitable organic dye material is available, which provides high resolution similar to recording materials used for writable optical disk, than it could be possible to design high density optical disk with the surface density close or more to Blu-ray disk.

Laser thermo-lithography, also known as phasetransition mastering transforms an inorganic photoresist layer from the initial state into a transformed state by laser beam heating [3]. Recording pits can be reached by removing the transformed areas with acid or alkaline solutions. Furthermore, the sizes of recording pits can overcome the diffraction limit, which allows the usage of a visible laser beam as a light source and reduces the cost of equipment [3].

At the same time, in high-density thermolithography data recording, the relevant process takes place only in the area where phase transition occurs. The dimensions of the area can be significantly smaller than the laser spot size, which makes it possible to increase the density of optical recording. The goal of research consists in creation of technology for high-density recording on thin films of photosensitive nanocomposite materials.

In this paper, we propose alternative method to make high density optical discs, using the thermolithography principle. In our proposal, we introduced a fine structure in the recorded optical effects in such a manner that more bits than one can be stored per location.

\section{Laser thermo-lithography data recording on organic nanocomposite films}

Thermo-lithography data recording requires using a proper recording medium with predefined characteristics. In this work, intra-ionic conjugated systems (dyes) characterized by intensive and selective absorption in the spectral region $390-410 \mathrm{~nm}$ were elaborated. We used 3-styryl bases derivatives of benzothiazole substituted at phenyl residue or at chromophore; 4-nullmethinemerocyanines derivatives of thiobarbitutric acid and 6-membered nitrogen- or oxygen-containing heterocycles; one naphtostyryl derivative dyes [4]. These dyes are able to be evaporated by $405 \mathrm{~nm}$ laser radiation. The nanocomposite films were created on base of organic positive photoresist that is also sensitive to $405 \mathrm{~nm}$ radiation and added synthesized dye with various concentrations. The 150-nm layer of nanocomposite film was deposited on glass substrates. The process in accord with the high-density data recording method is close to mastering the optical discs for long-term data storage (Fig. 2) [5].

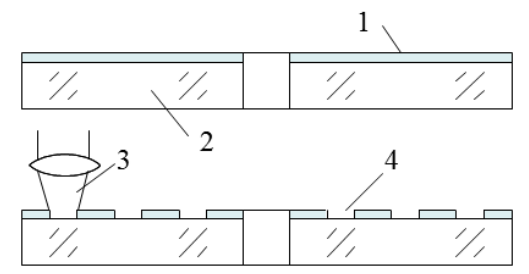

Nanocomposite film deposition

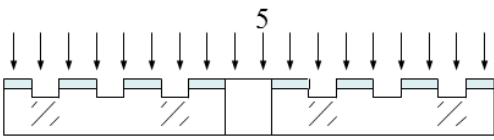

Data recording by focused laser beam

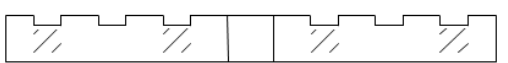

Removal of recording layer rests

Fig. 2. Stages of the data recording process by laser thermolithography and reactive ion-beam etching: 1 - nanocomposite film, 2 - substrate, 3 - focused laser beam, 4 - pits in nanocomposite films, 5 - ion beam. 
During the recording process, the nanocomposite film layer was heated and particularly evaporated under the influence of laser radiation. The concentration of dye in the film defines intensity of light absorption and temperature gradient within the layer. This gradient should be as sharp as possible in order to get a minimum characteristic size of obtained pits. Note that pit profile is defined by decomposition temperature of the film as well. Thermogravimetric analysis of several nanocomposite films based on various organic dyes with different decomposition temperature showed that thermal properties of the film are mainly defined by photoresist properties. The analysis showed that decomposition temperature of photoresist is close to $540{ }^{\circ} \mathrm{C}$. The minimum pit width was reached using organic dye with $270{ }^{\circ} \mathrm{C}$ decomposition temperature and 0.6 mass $\%$ concentration in the film.

In the result, the pits with $250-300-n m$ width were obtained on the thin organic nanocomposite film by using master-disc laser-burning station with 405-nm laser beam focused with 0.85 NA lens. It should be noted that conventional photochemical data recoding on this laser-burning station allows obtaining only 500 - $550 \mathrm{~nm}$ pits widths [6]. Another difference of the obtained pits is its tapered form with $50-100-\mathrm{nm}$ bottoms (Fig. 3). This allows to use the obtained pits as a mask for subsequent reactive ion-beam etching because the bottom size will defined final pits size.

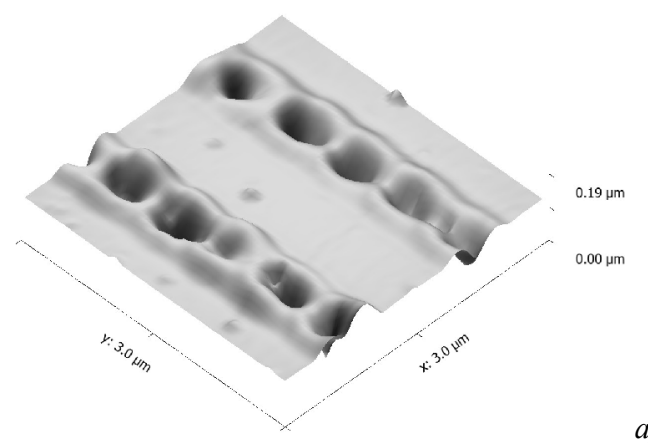

a)

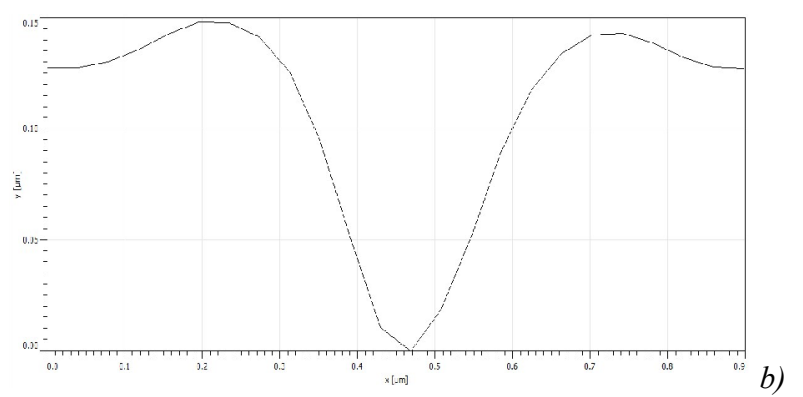

Fig. 3. Nanocomposite film surface after recording (a) and initial pit profile (b).

\section{Final pit formation with ion-beam etching}

For high-density data recording, it is necessary to apply dry etching techniques based on sputtering the substrate material atoms by high energy ions of gas-discharge plasma [7]. Ion-beam etching has highest anisotropy among these methods because material etching is executed by directional bombardment by inert gas plasma ions. In this case, the undercutting of structures sidewall is insignificant. It allows creating nanostructures with 0.01 cross size accuracy of the structure depth.

Reactive ion-beam etching is used to increase the etching rate and selectivity [7]. It is based on application of reactive gases in contrast to the previous one. In this case, the etching is based on reciprocally amplified processes of impact interaction and chemical reactions between plasma ions and work material atoms. It sufficiently increases etch speed and selectivity, but keeps a high level of anisotropy.

The nanocomposite film with obtained pits was used as a mask for reactive ion-beam etching of soda-siliceous glass substrate. Operating $\mathrm{CF}_{4}$ gas is usually used for siliceous materials [7]. Then, we also use this gas for reactive ion-beam etching of soda-siliceous glass substrate surface. The $150-200-\mathrm{nm}$ pits were obtained on the substrate surface in the result of the etching (Fig. 4). The size of obtained pits is close to that of Blu-ray format. Then, the proposed method may be considered as an alternative way to create master-discs for BD media.

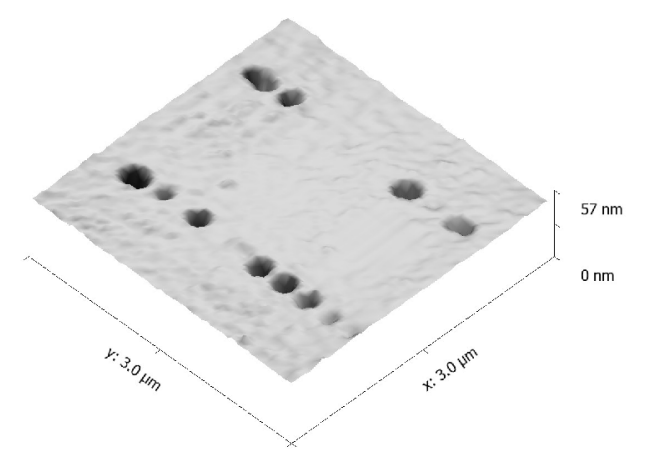

a)

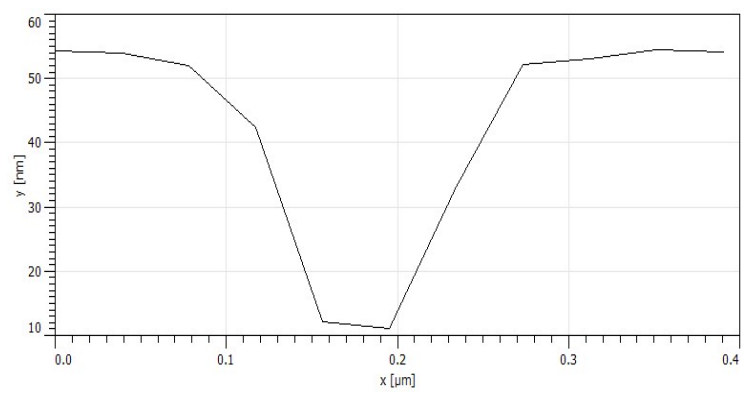

b)

Fig. 4. Substrate surface after ion-beam etching (a) and the final pit profile (b). 


\section{Conclusions}

Proposed nanocomposite organic films allows switching data recoding process from conventional photochemical to laser thermo-lithography that considerably decreased pits sizes from 550 down to $300 \mathrm{~nm}$. The proposed ionbeam etching additionally decreased pit sizes down to $150-200 \mathrm{~nm}$. The discussed laser thermo-lithography method may be used as an alternative way to create master-discs for Blu-ray media. The proposed method may be used to fabricate high-density optical media for long-term data storage as well.

\section{References}

1. E. Meinders, R. Rastogi, M. van der Verr, P. Peeters, H. Majdoubi, H. Bulle, A. Millet, D. Bruls. Phase transitions mastering of high-density optical media // Japanese Journal of Applied Physics, 46 (6B), p. 3987-3992 (2007).

2. A. van de Nes, J. Braat, S. Pereira. High-density optical data storage // Reports on Progress in Physics, 69, p. 2323-2363 (2006).

3. Jh. Chen, J. Lin, J. Chen, K. Chiu. Optimization of Ge-Sb-Sn-O Films for Thermal Lithography of
Submicron Structures // Japanese Journal of Applied Physics. 51, p. 06FC03-1 - 06FC03-6. DOI: 10.1143/JJAP.51.06FC03 (2012).

4. K. Grytsenko, O. Belyaev, A. Kryuchin, I. Gorbov, S. Schrader, V. Ksianzou. Optical recording media based on nanoparticles for superhigh density information storage // Optical Memory and Neural Networks, 22(3), p. 127-134 (2013).

5. V. Petrov, A. Kryuchyn, I. Gorbov. High-density optical disks for long-term information storage // Proc. SPIE 8011, 22nd Congress of the International Commission for Optics: Light for the Development of the World, 80112J, DOI: $10.1117 / 12.900745$ (25 October 2011).

6. V.V. Petrov, A.A. Kryuchyn, S.M. Shanoylo, V.G. Kravets, I.O. Kossko, E.V. Belyak, A.S. Lapchuk, S.A. Kostyukevych Super-dense optical registrtation of information - National Academy of Sciences of Ukraine, Institute for Information Recording. - Kiev: National Academy of Sciences of Ukraine, 2009.$282 \mathrm{p}$.

7. V.V. Petrov, A.A. Kryuchyn, I.V. Gorbov. Using ion beams for crea-tion of nanostructures on the surface of high-stable materials // Semiconductor Physics, Quantum Electronics \& Optoelectronics, 10(1), p. 27-29 (2007). 